\title{
Silicon Sensor and Detector Developments for the CMS Tracker Upgrade
}

\author{
Raffaello D'Alessandro*i \\ Università degli Studi di Firenze and INFN Sezione di Firenze \\ E-mail: candiefi.infn.it
}

CMS has started a campaign to identify the future silicon sensor technology baseline for a new Tracker for the high-luminosity phase of LHC, coupled to a new effective way of providing tracking information to the experiment trigger. To this end a large variety of 6" wafers was acquired with different thicknesses and crystal material from HPK and new detector module designs were investigated. Detector thicknesses ranging from $50 \mu \mathrm{m}$ to $300 \mu \mathrm{m}$ are under investigation on Float Zone, Magnetic Czochralski and Epitaxial material both in n-in-p and p-in-n versions. P-stop and p-spray are explored as isolation technology for the n-in-p type sensors as well as the feasibility of double metal routing on 6" wafers. Each wafer contains different structures to answer different questions, e.g. influence of geometry, Lorentz angle, radiation tolerance, annealing behaviour, validation of read-out schemes. Dedicated process test-structures, as well as diodes, mini-sensors, long and very short strip sensors and real pixel sensors have been designed for this evaluation. The structures will be exposed to fluences composed of protons and neutrons representing the mixture of charged hadrons and neutrons as expected in the CMS Tracker after an integrated luminosity of $3000 \mathrm{fb}^{-1}$ at several radii. At the same time simulation studies were performed on how identification of high-momentum particle tracks, as a part of the Level 1 Trigger, can be achieved by correlating hits on two closely spaced sensors (stacked modules), making use of their strip or pixel cluster widths and positions to estimate the track bending in the high CMS magnetic field. This contribution provides an overview of the individual sensor structures and their characteristics and of the construction of different types of stacked modules for trigger studies, and summarizes the interesting measurements performed so far.

The 2011 Europhysics Conference on High Energy Physics, EPS-HEP 2011,

July 21-27, 2011

Grenoble, Rhône-Alpes, France

*Speaker.
'On behalf of the CMS Tracker Collaboration. 


\section{Introduction}

The CMS Tracker for SLHC needs to have higher radiation resistance with respect to both instantaneous and integrated levels of luminosity, and higher readout granularity to keep the channel occupancy at an adequate level. An eloquent example of the problems that will have to be met at sLHC is shown in Fig.1. Also a new development on Tracker detector modules and sensors has started in order to be able to give information about track pT and direction to the first level trigger so as to reduce the number of low energy particle tracks in the trigger processing. Thus
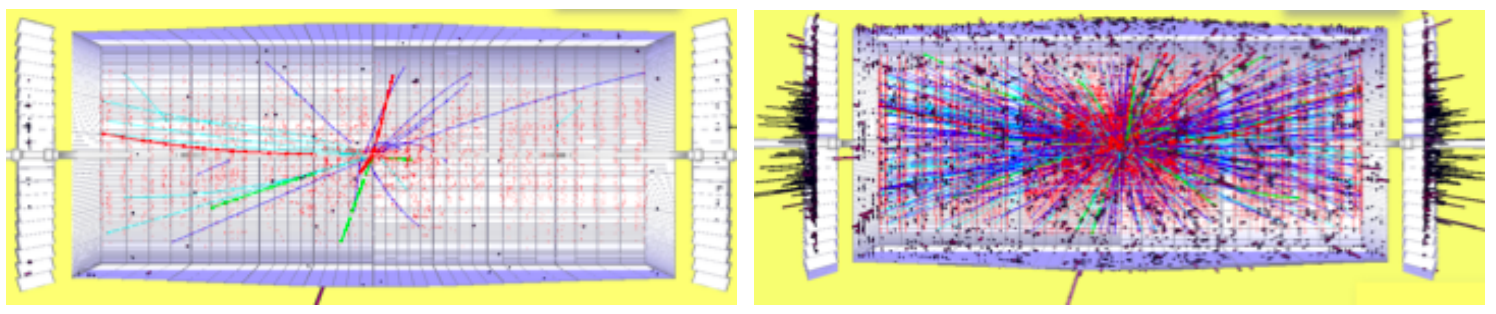

Figure 1: Event multiplicity example. At the left at $10^{33} \mathrm{~cm}^{-2} \mathrm{~s}^{-1}$ (the current LHC luminosity), at right at $10^{35} \mathrm{~cm}^{-2} \mathrm{~s}^{-1}$, the sLHC envisaged luminosity.

the challenge facing sLHC trackers can be summarised as such: build a replacement Tracker for L $>10^{34} \mathrm{~cm}^{-2} \mathrm{~s}^{-1}$ with $\mathrm{L} 1$ trigger capabilities. The silicon sensors will have to survive accumulated dose levels 10 times higher than current Tracker and have higher granularity. With this purpose CMS $[1,2]$ has undertaken an extensive R\&D focusing on innovative sensor substrates and technologies, such as thin sensors, epitaxial growth and the likes. In this paper the focus will be on strip detector $R \& D$ which will continue to populate the outer layers $(R>50 \mathrm{~cm})$ of the future Tracker at sLHC. The objective is then to conduct tests, before and after irradiation, to determine the characteristics of single-sided silicon sensors of various thicknesses and materials in order to establish optimal material and strip/pixel features for the upgrade of the CMS Tracker.

\section{Silicon sensor types}

\begin{tabular}{lcccccc}
\hline Region & 1 & 2 & 3 & 4 & 5 & 6 \\
\hline Sensor Label & $1-120$ & $1-240$ & $1-80$ & $1-70$ & $2-120$ & $2-240$ \\
\hline Pitch $(\mu \mathrm{m})$ & 120 & 240 & 80 & 70 & 120 & 240 \\
Implant Width $(\mu \mathrm{m})$ & 16 & 34 & 10 & 8.5 & 28 & 58 \\
Metal Width $(\mu \mathrm{m})$ & 29 & 47 & 23 & 21.5 & 41 & 71 \\
Width/Pitch & 0.133 & 0.142 & 0.125 & 0.121 & 0.233 & 0.242 \\
\hline
\end{tabular}

Table 1: Main parameters of the first set of six region types under investigation for radiation hardness.

Many sensor types have been produced. In particular a multi geometrical sensor consisting of 12 regions with different geometrical characteristics has been produced in many substrate flavour types, such as: FloatZone (FZ), Magnetic - Czochralski (MCZ), Epitaxial (Epi); also the implants have been realised in different technologies, $\mathrm{p}$-in-n (N-type), n-in-p with p-stops (P-type), n-in-p with p-spray (Y-type), p-in-n (double metal), n-in-p (p-stop, double metal), n-in-p (p-spray, double 
metal). The outer dimensions of the sensor are $65744 \mu \mathrm{m} \times 32792 \mu \mathrm{m}$ and the 12 regions characteristics are shown in table 1 and 2. The production mask for the wafer and a mounted MSSD

\begin{tabular}{lllllll}
\hline Region & 7 & \multicolumn{1}{c}{8} & 9 & 10 & 11 & 12 \\
\hline Sensor Label & $2-80$ & $2-70$ & $3-120$ & $3-240$ & $3-80$ & $3-70$ \\
\hline Pitch $(\mu \mathrm{m})$ & 80 & 70 & 120 & 240 & 80 & 70 \\
Implant Width $(\mu \mathrm{m})$ & 18 & 15.5 & 40 & 82 & 26 & 22.5 \\
Metal Width $(\mu \mathrm{m})$ & 31 & 28.5 & 53 & 95 & 39 & 35.5 \\
Width/Pitch & 0.225 & 0.221 & 0.333 & 0.342 & 0.325 & 0.321 \\
\hline
\end{tabular}

Table 2: Main parameters of the second set of six region types under investigation for radiation hardness.

detector are shown in Figure 2. The MSSD structure is glued to a ceramic frame and bonded to a pitch adapter. The ensemble can then be bonded to a connectorised PCBs (as in the picture) or to a FE hybrid for particle detection measurements in the laboratory or at test beams. The structure shown is ready to be placed inside a custom made climatic chamber that can reach $-20^{\circ} \mathrm{C}$. The connectors attached allow to perform current vs voltage (IV) measurements and capacitance vs voltage $(\mathrm{CV})$ measurements. The capacitance is measured both from strip to backplane and from strip to neighbouring strips.
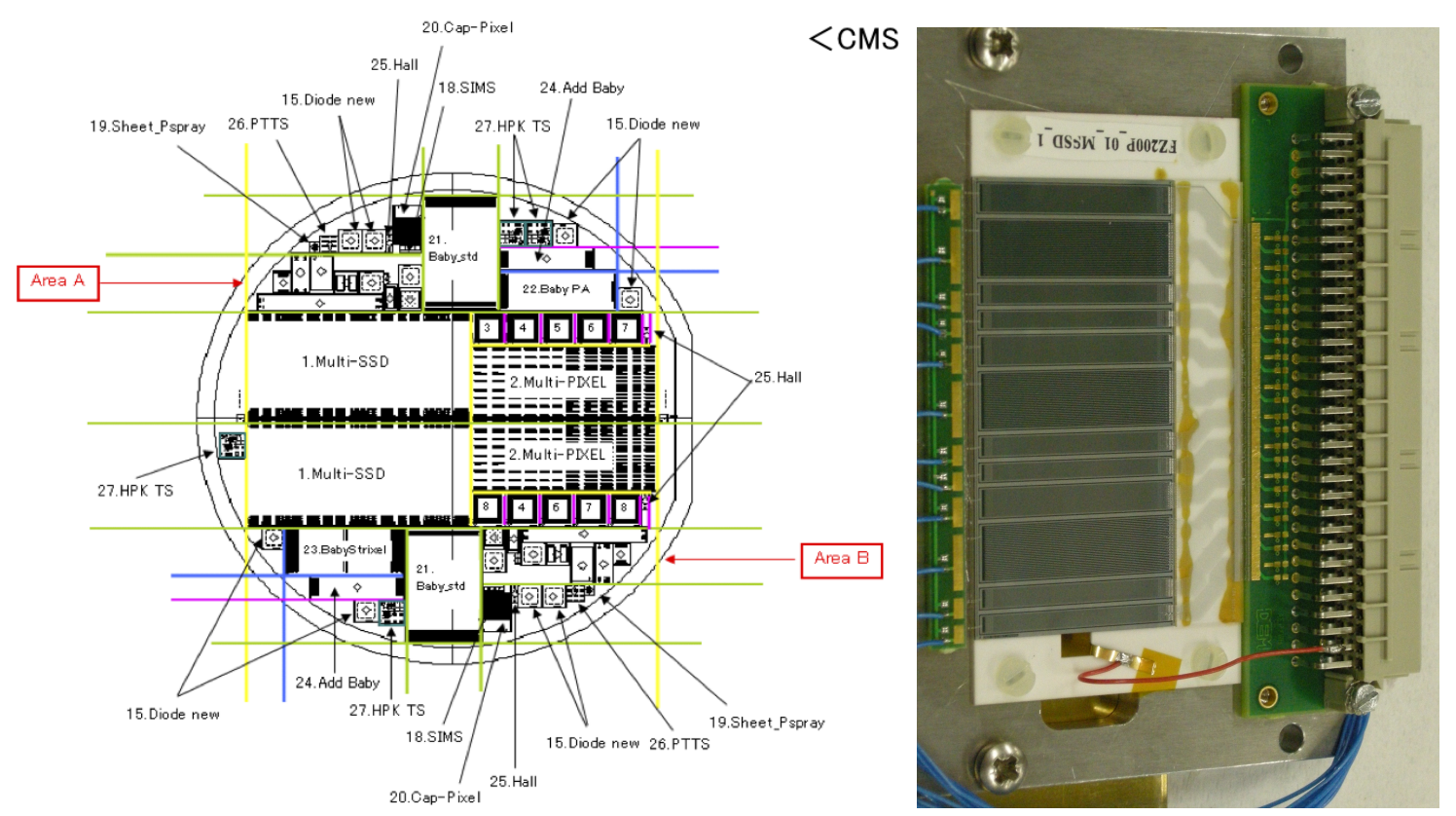

Figure 2: The sensor wafer mask (left). Amongst the many regions (pixels, strixels, etc.) there are two large rectangular regions which are the multigeometry strip detectors (MSSD). At left a mounted MSSD structure with the 12 different regions clearly visible, ready for testing.

\section{First measurements}

The MSSD campaign has just started. In Florence more than a hundred structures were glued 


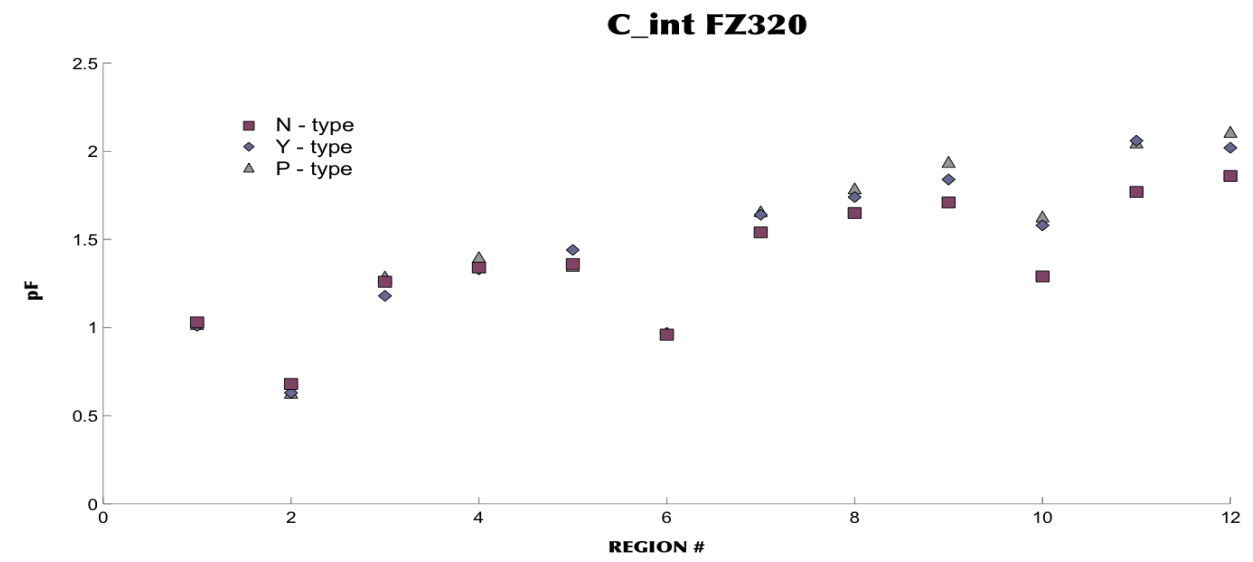

Figure 3: The Cint (strip to strip) capacitance as measured for the 12 regions of FZ sensors of 320 micron thickness, with different implant technology.

and bonded. As the structures became available CV and IV measurement were performed at $20^{\circ} \mathrm{C}$. Results on unirradiated FZ sensors for Cint (strip to strip) capacitance are shown in Fig. 3.

The collaboration has now started a systematic campaign of characterizations and has also started the first irradiations with neutrons and protons. First comprehensive results should become available later this year, but the program will last at least till the end of 2012.

\section{Trigger capable silicon detectors}

The idea is to derive locally transverse momenta information from the measurement of the track inclination [3] either through a cluster width algorithm or using two sensors one on top of the other separated by a 1-2 mm spacer (first part of Fig.4). In the first case, track selection is achieved by counting the number of strips above threshold belonging to a cluster (this number is anti-correlated to the transverse momentum of the track), while in the second track inclination is derived directly from the two modules cluster positions. FE electronics must be capable of reading one or two sensors, depending on the chosen approach, and should provide filtering, transmitting only high $\mathrm{p}_{\mathrm{T}}$ tracks data to the trigger processors. A first idea of the attainable performance was
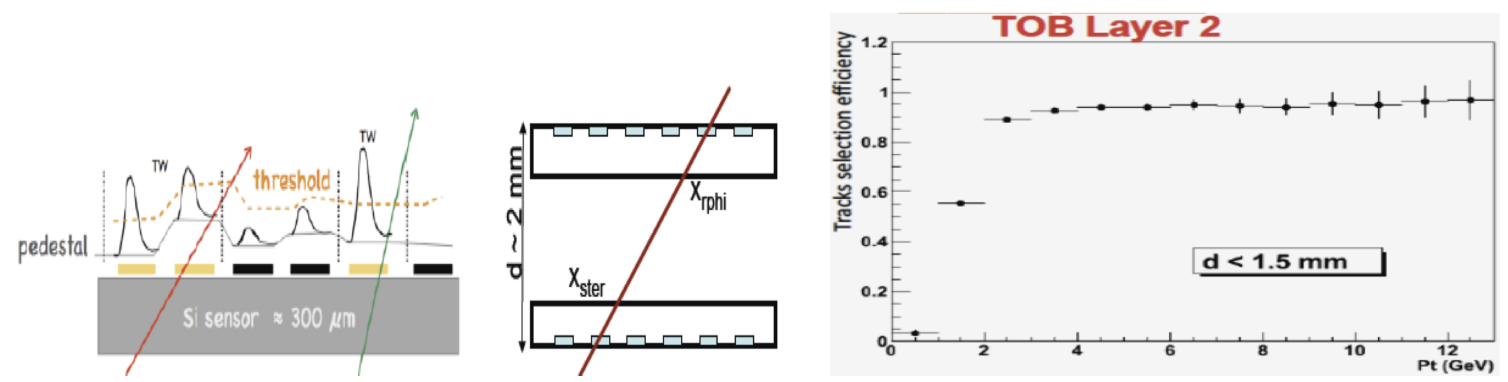

Figure 4: To the left, a schematic drawing of the concept behind $\mathrm{p}_{\mathrm{T}}$ suppression. (I) refers to the cluster width method (CW), (II) to the stacked detector concept. At the right, the turn on efficiency for tracks as a function of $\mathrm{p}_{\mathrm{T}}$. The method tested relies on local track inclination measurement. Tracks below $2 \mathrm{GeV} / \mathrm{c}$ are suppressed. 
studied using the current CMS Tracker with real data. For example the turn on efficiency for a two layer module is shown in Fig.4. In this case a double sided module of the current Tracker was chosen (separation between the two sensors of about $1.5 \mathrm{~mm}$ ).

\section{Conclusions}

A very thorough and comprehensive R\&D campaign has been undertaken by the CMS collaboration in order to find viable solutions for a sLHC Tracker. One of the main items is the silicon sensor technology to be used. In the next years the sensor R\&D will provide needed inputs to the design of the new Tracker.

\section{References}

[1] CMS Expression of Interest in the SLHC, published in CERN/LHCC 2007-014, LHCC-G-131, 15 March 2007 .

[2] CMS Tracker upgrade, published in PoS VERTEX2009:034, 2009.

[3] Concepts for a Tracker trigger based on a multi-layer layout and on-detector data reduction using a cluster size approach, published in JINST 5:C08002, 2010 . 